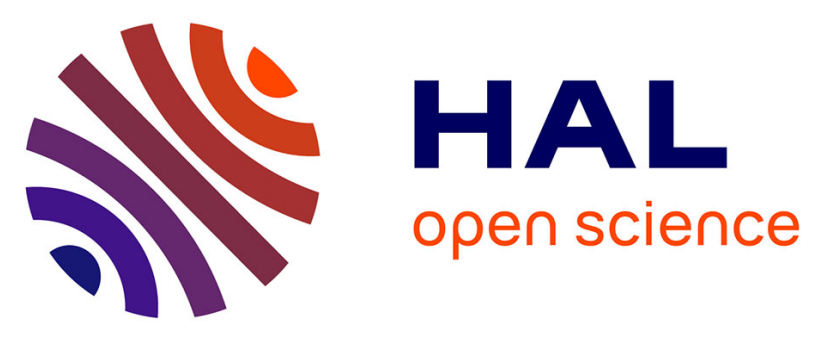

\title{
Discrimination of deletion to point cytokine mutants based on an array of cross-reactive receptors mimicking protein recognition by heparan sulfate
}

Maria Genua, Laurie-Amandine Garçon, Yulia Sergeeva, Els Saesen, Benjamin Musnier, Arnaud Buhot, Martial Billon, Evelyne Gout, Rabia Sadir, Hugues Lortat-Jacob, et al.

\section{To cite this version:}

Maria Genua, Laurie-Amandine Garçon, Yulia Sergeeva, Els Saesen, Benjamin Musnier, et al.. Discrimination of deletion to point cytokine mutants based on an array of cross-reactive receptors mimicking protein recognition by heparan sulfate. Analytical and Bioanalytical Chemistry, 2022, 414, pp.551-559. 10.1007/s00216-021-03516-z . hal-03340313

\section{HAL Id: hal-03340313 https://hal.science/hal-03340313}

Submitted on 13 Oct 2021

HAL is a multi-disciplinary open access archive for the deposit and dissemination of scientific research documents, whether they are published or not. The documents may come from teaching and research institutions in France or abroad, or from public or private research centers.
L'archive ouverte pluridisciplinaire HAL, est destinée au dépôt et à la diffusion de documents scientifiques de niveau recherche, publiés ou non, émanant des établissements d'enseignement et de recherche français ou étrangers, des laboratoires publics ou privés. 


\title{
Discrimination of Deletion to Point Cytokine Mutants Based on an Array of Cross-Reactive Receptors Mimicking Protein Recognition by Heparan Sulfate
}

\author{
Maria Genua, ${ }^{1}$ Laurie-Amandine Garçon, ${ }^{1}$ Yulia N. Sergeeva, ${ }^{1}$ Els Saesen, ${ }^{2}$ Benjamin Musnier, ${ }^{1}$ Arnaud Buhot, ${ }^{1}$ \\ Martial Billon, ${ }^{1}$ Evelyne Gout, ${ }^{2}$ Rabia Sadir, ${ }^{2}$ Hugues Lortat-Jacob, ${ }^{2}$ Christine Le Narvor, ${ }^{3}$ David Bonnaffé, ${ }^{3}$ \\ Thierry Livache, ${ }^{1}$ Yanxia Hou ${ }^{1, *}$ \\ ${ }^{1}$ Université Grenoble Alpes, CEA, CNRS, IRIG-SyMMES, 38000 Grenoble, France \\ ${ }^{2}$ Institut de Biologie Structurale, UMR 5075, Université Grenoble Alpes, CNRS, CEA, 38000 Grenoble, France \\ ${ }^{3}$ Université Paris-Saclay, CNRS, Institut de chimie moléculaire et des matériaux d'Orsay, 91405, Orsay, France \\ Corresponding author: Yanxia Hou, email: Yanxia.hou-broutin@cea.fr
}

\begin{abstract}
Differential sensing of proteins based on cross-reactive arrays and pattern recognition is a promising technique for the detection and identification of proteins. In this study, a rational biomimetic strategy has been used to prepare sensing materials capable of discriminating structurally similar proteins, such as deletion and point mutants of a cytokine, by mimicking the biological properties of heparan sulfate (HS). Using the self-assembly of two disaccharides, lactose and sulfated lactose at various ratios on the surface of a chip, an array of combinatorial cross-reactive receptors has been prepared. Coupling with surface plasmon resonance imaging (SPRi), the obtained cross-reactive array is very efficient for protein sensing. It is able to detect HS binding proteins (HSbps) such as IFN $\gamma$ at nanomolar concentrations. Moreover, such a system is capable of discriminating between IFN $\gamma$ and its mutants with good selectivity.
\end{abstract}

\section{Key words:}

Heparan sulfate, Interferon- $\gamma$, Cross-reactive, Pattern recognition, Surface plasmon resonance imaging

\section{Introduction}

Differential sensing of proteins based on cross-reactive arrays and pattern recognition is a promising alternative to the system based on 'lock-and-key' recognition principle such as biosensors for the detection and identification of proteins [1] and for biomedical applications [2]. This approach takes inspiration from the mammalian olfactory system, which uses cross-reactive receptors to create a pattern or a sample fingerprint. Thereby, it overcomes the difficulties associated with design and synthesis of highly selective receptors. In the last two decades, many different strategies have been developed to achieve high performance synthetic receptors or functionalized nanoparticles for differential sensing of proteins,[3] such as tetra-carboxyphenylporphyrin coupled to different amino 
acids or dipeptides on their periphery developed by Hamilton and co-workers, [4, 5] synthetic scaffolds consisting of an hexa-substituted benzene core combined with two tripeptide arms selected using combinatorial chemistry designed by Anslyn's group,[6] poly(p-phenyleneethynylene)s (PPEs) derivatives [7] and later on gold nanoparticle conjugates developed by Rotello's group, [8-10] ruthenium(II) tris(bipyridyl) protein surface mimetics by Hewitt and Wilson,[11] dual-ligand cofunctionalized fluorescent gold nanodots by Yuan et al.,[12] ionic liquids or their conjugates with quantum dots by Chen et al.,[13] blue-emitting fluorescent gold nanoclusters by Xu et al.,[14] near infrared fluorescent dual ligand functionalized gold nanoclusters designed by Luo's group,[15, 16] aptamer-functionalized gold nanoparticles by Zhang's group [17] and some other colorimetric sensor array based on gold nanoparticles summarized in the review [18]. Based on these sensing materials, the obtained cross-reactive arrays were very efficient in detecting, identifying, and quantifying pure proteins at the low nanomolar concentration range [8] or to discriminate between a mixture of different proteins in human serum at physiological relevant concentrations.[9] Nevertheless, two major challenges remain: the production of sensing receptors with good selectivity for distinguishing between structurally similar analytes and the development of a single, label free, and reusable analytical device that allows monitoring of binding events in real-time.

In this study, we aim to develop a rational biomimetic methodology for preparing sensing receptors for the analysis of HSbps, inspired by the way HS polysaccharide recognizes its ligand, and to develop a reusable microarray that allows good discrimination between proteins featuring similar structures, such as deletion and point mutants.

HS, a large anionic polysaccharide, belongs to the family of glycosaminoglycan (GAG) that is widely present in the extracellular matrix and at the cell surface. It interacts with a multitude of HS binding proteins, including cytokines, chemokines, growth factors/morphogens and their receptors, etc, and thus has crucial regulatory roles in many biological processes.[19] It is crucially relevant to many disease processes, including inflammation,[20] angiogenesis,[21] neurodegeneration,[22] and cancer.[23] This makes HS mimicry an attractive subject for the design of new efficient drugs or novel sensing methods targeting HSbps. Therefore, HS and their interaction with HSbps represents a very interesting model for our study.

HS is one of the most heterogeneous biopolymers with two levels of molecular diversity.[24, 25] The first level corresponds to the micro-heterogeneities resulting from the variation in sulfation and epimerization of its constituting disaccharide building blocks (glucuronic/iduronic acid - glucosamine). In addition, the HS chain can be divided into three domains: A domains, which are mainly composed of D-glucuronic acid and $\mathrm{N}$-acetylated D-glucosamine and thus have low charge density; $\mathrm{S}$ domains, which are rich in L-iduronic acid and $\mathrm{N}$-sulfated D-glucosamine and highly charged; mixed $\mathrm{A} / \mathrm{S}$ regions of variable length make the transition between $\mathrm{A}$ and $\mathrm{S}$ domains. Taken together, the various combinations of S, A and A/S domains generate multiple SAS charge topologies along the polymer chain and represent the second level of molecular diversity, as illustrated in Scheme 1a. Depending on 
cell type and activation state, HS chains with different negatively charged topologies are expressed, presumably promoting selective interactions with HSbps.[26, 27]

Recent studies reveal that many protein-HS interactions depend primarily on the overall charge density and distribution, rather than on the distinct saccharide sequence and the fine structure (specific sulfation and epimerization).[28, 29] Therefore, herein, we intended to mimic the biological properties of HS with a cross-reactive array, prepared by self-assembly of a small number of disaccharides used as building blocks (BBs) with different physicochemical properties on a surface of a chip. For this proof-of-concept study, two disaccharides neutral lactose (BB1) and negatively charged sulfated lactose (BB2) were used (Scheme 1b). We assumed that mixing these two molecules at different ratios should generate, following their self-assembly on the surface of a chip, various combinatorial surfaces with different charge densities and topologies. Each surface can be considered as a combinatorial cross-reactive receptor (CoCRR), which provides the micro-heterogeneities, just like the first level of diversity in HS. If such a biomimetic strategy works, more BBs with complementary physicochemical properties could be added in the CoCRR array to better mimic the biological properties of HS.

Herein, the efficiency of such a CoCRR array for protein sensing is evaluated using the cytokine interferon- $\gamma$ (IFN $\gamma$ ) together with six mutants displaying a range of modification level. IFN $\gamma$ strongly interacts with HS and plays an important role in modulating most phases of the inflammatory and immune response.[30] For protein sensing, the CoCRR array was coupled to a SPRi optical detection system. SPRi measures modifications of the refractive index at the surface of the chip. The binding of the protein on the CoCRRs induces a shift of the plasmon curves and thus a variation of reflectivity. In contrast to commonly used colorimetric and fluorescent methods,[4-10] which require preparation of an array per sample, and high consumption of protein since the analysis needs to channel each protein sample to different spatially separated receptors, SPRi fits perfectly for protein sensing. Thanks to imaging mode, it allows for a label-free, parallel and synchronous observation of binding events over the whole array in a multiplex format.[31, 32] The system is reusable after regeneration. More importantly, SPRi is able to monitor binding events in real-time, providing important information on the adsorption and desorption kinetics.[33, 34] Therefore, it can provide supplementary discrimination parameters since similar affinities can result in different kinetics.

\section{Materials and Methods}

\section{Materials}

The two disaccharides (Scheme 1) lactose (BB1) and sulfated lactose (BB2) were prepared from a $\beta$ lactoside, which was first converted to lactosylated disulfide BB1. Then, sulfatation was performed to obtain the fully sulfated disulfide BB2. The detailed synthesis of the disaccharides was reported previously.[35]

Sodium chloride, sodium dihydrogen phosphate, HEPES, sodium dodecyl sulphate (SDS), Tween 20 and glycerol were purchased from Sigma-Aldrich. All of them were used as received. Protein lectin 
from Erythrina cristagalli (ECL), bovine serum albumin (BSA), Immunoglobulin G (IgG) from rabbit serum, and Cytochrome $\mathrm{C}(\mathrm{CytC})$ from horse heart were also purchased from Sigma-Aldrich and used as received. In this study, ultrapure water $(18.2 \mathrm{M} \Omega . \mathrm{cm})$ used for preparation of buffer solutions was produced by a Purelab classic system (Elga).

\section{IFN $\gamma$ wild type and mutant expression and purification}

Human IFN $\gamma$ cDNA (a kind gift from Jean-Hervé Colle, Pasteur Institut) was cloned into pET11a expression vector (Novagen), and used to transform E. coli strain BL21 Star DE3 (Invitrogen).[36, 37] Cells were grown at $37^{\circ} \mathrm{C}$ in Luria broth medium containing $100 \mu \mathrm{g} / \mathrm{mL}$ ampicillin, and induced with $0.5 \mathrm{mM}$ IPTG for $5 \mathrm{~h}$. Purification from inclusion bodies (IB) was then performed. Briefly, for IFN $\gamma$ $\mathrm{SD} 1, \mathrm{SD} 2, \Delta 136$ and $\Delta 124$, IB were solubilized in $6 \mathrm{M}$ guanidine $\mathrm{HCl}$ at $10 \mathrm{mg} / \mathrm{mL}$, after which the protein was refolded by dilution to $0.36 \mathrm{mg} / \mathrm{mL}$ into $100 \mathrm{mM}$ Tris- $\mathrm{HCl}$ buffer at $\mathrm{pH} 8$ containing, 0.5 M L-Arg, $0.2 \mathrm{mM}$ EDTA and protease inhibitors. Samples were then dialyzed against $20 \mathrm{mM}$ Tris$\mathrm{HCl} \mathrm{pH}$ 8.0, containing $20 \mathrm{mM}$ urea. For IFN $\gamma \mathrm{S} 129$ and S130, IB were also solubilized in $6 \mathrm{M}$ $\mathrm{Gdn} / \mathrm{HCl}$ but at $2.4 \mathrm{mg} / \mathrm{mL}$ and refolded by dilution to $0.2 \mathrm{mg} / \mathrm{mL}$ into $50 \mathrm{mM}$ phosphate buffer and $0.5 \mathrm{M} \mathrm{Gdn} / \mathrm{HCl} \mathrm{pH} \mathrm{7,} \mathrm{supplemented} \mathrm{with} \mathrm{protease} \mathrm{inhibitors.}$

The IFN $\gamma$ samples were centrifuged for $20 \mathrm{~min}$ at $10000 \mathrm{rpm}$, and the supernatants were subsequently brought to $\mathrm{pH}$ 6.8. Wild-type and mutant IFN $\gamma$ were then purified by ion exchange chromatography (Source S HR 5/5 column in $50 \mathrm{mM}$ phosphate buffer, pH 6.8) and gel filtration (Superdex 75 column equilibrated in $10 \mathrm{mM}$ Tris and $10 \mathrm{mg} / \mathrm{mL}$ mannitol, $\mathrm{pH}$ 6.8).

\section{Preparation of the combinatorial cross-reactive receptor array}

For this, the pure and mixed solutions of BB1 and BB2 were deposited on gold surface of a prism, provided by Horiba Scientific (Orsay, France). Forty-eight hours prior to spotting, the prism was treated by plasma (0.6 mbar, 75\% Oxygen, 25\% Argon, power $40 \mathrm{~W}, 3 \mathrm{~min}$ ) with a Femto plasma cleaner (Diener Electronic, Germany). Afterwards, eleven solutions with [BB1]/([BB1]+[BB2]) ratios $(0,10,20,30,40,50,60,70,80,90$ and $100 \%)$ were prepared in PBS $\left(50 \mathrm{mM} \mathrm{NaH} \mathrm{PO}_{4}, 150 \mathrm{mM}\right.$ $\mathrm{NaCl}, 10 \%$ glycerol, $\mathrm{pH} 6.8$ ) with a total disaccharide concentration at $0.1 \mathrm{mM}$. Then these solutions were spotted at a volume of $8 \mathrm{~nL}$ using a non-contact spotter (sciFLEXARRAYER S3, Germany). For each ratio, four replicates were made and placed randomly on the prism. The prism was then left overnight at room temperature for the formation of CoCRRs via self-assembly. Notably, the addition of $10 \%$ glycerol in PBS minimized the evaporation of water in the spots during this self-assembly process and thus guaranteed a good reproducibility. Finally, the prism was cleaned thoroughly with ethanol and ultrapure water and dried under a flow of $\mathrm{N}_{2}$. It was kept at $4{ }^{\circ} \mathrm{C}$ in refrigerator before use.

\section{Protein sensing by surface plasmon resonance imaging}

Protein sensing was performed at $25^{\circ} \mathrm{C}$ with a SPRi apparatus (Horiba Scientific), which was put in a temperature regulated incubator (Memmert, Germany). A collimated beam from a LED was polarized and sent towards the functionalized gold surface through the prism to illuminate the entire microarray. 
The intensity of the reflected light was monitored by a CCD camera. In this study, SPRi measurements were performed at a fixed working angle, chosen at the highest slope of the plasmon curves.

The functionalized prism was placed in a PEEK flow cell with a volume of $10 \mu \mathrm{L}$ and a hexagonal configuration. The flow cell was connected to a degassing system (Alltech, France), a 6-port medium pressure injection valve (Upchurch Scientific, USA) for sample injection, and a computer controlled syringe pump (Cavro XLP 6000, Cavro scientific instruments, USA).

In this study, freshly filtered and degassed HEPES solution $(10 \mathrm{mM}, \mathrm{pH} 7.4)$ was used as running buffer solution for performing SPRi experiments. Protein samples were also prepared using this buffer and then injected onto the prism with a volume of $500 \mu \mathrm{L}$ at a $100 \mu \mathrm{L} / \mathrm{min}$ flow rate ( 5 min infusion). Once injected, molecular binding occurred. Consequently, all the spots of the cross-reactive receptors lighted up with different intensities according to their affinity with the protein. This binding event was registered using an 8-bit CCD video camera at a fixed interval of time $(0.2 \mathrm{~s})$. After each injection, the array was rinsed with running buffer at a $100 \mu \mathrm{L} / \mathrm{min}$ flow rate and then regenerated with $2 \%$ SDS $(\mathrm{w} / \mathrm{w})$ dissolved in ultrapure water for reuse. At least three replicated injections and measurements were performed in a random order for each protein sample on the same CoCRR array.

Moreover, a reference sample, protein ECL at $200 \mathrm{nM}$ was used as a standard to evaluate the functionality of the $\mathrm{eT}$ in terms of stability and repeatability.[33] It was systematically injected onto the CoCRR array at the beginning before the injection of protein of interest, several times randomly in the middle and at the end of all protein sensing.

\section{Data processing}

SPRi monitors the changes of the refractive index taking place at the vicinity of the surface of the CoCRRs upon protein injection using the CCD video camera, which registers real-time images across the whole array. Using Imaging software (Horiba Scientific), all SPR images were converted to variations of reflectivity (expressed as $\mathrm{R} \%$ ) versus time and thus generated a series of kinetic binding curves, called sensorgrams. By plotting the response $(\mathrm{R} \%)$ of each combinatorial surface for the protein sample at the end of injection versus BB1\%, a 2D continuous evolution profile (CEP) was generated. By adding the BB1\% in sensorgrams, 3D continuous evolution landscape (CEL) was generated for protein sample, which allows to integrating adsorption and desorption kinetics.

\section{Results and Discussion}


a)

$1^{\text {st }}$ level of diversity: micro-heterogeneities

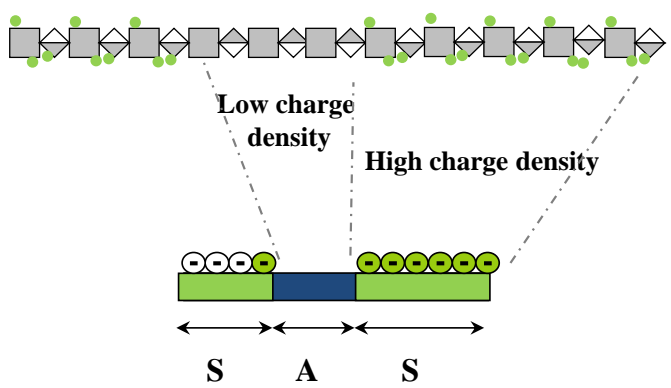

$2^{\text {nd }}$ level of diversity: charge topology

$\mathrm{N}$-acetylated/sulfated glucosamine

$\diamond$ Glucuronic acid

$\diamond$ Iduronic Acid

Sulfate b)

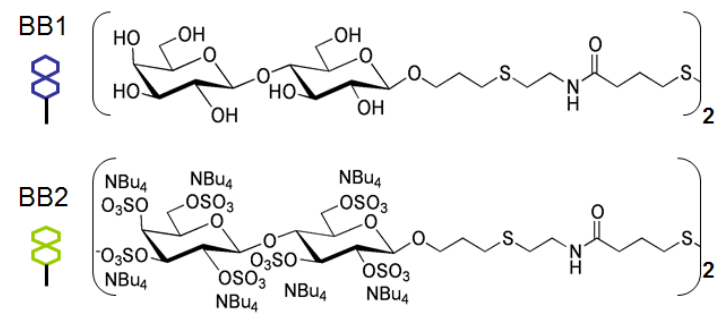

High charge density Low charge density

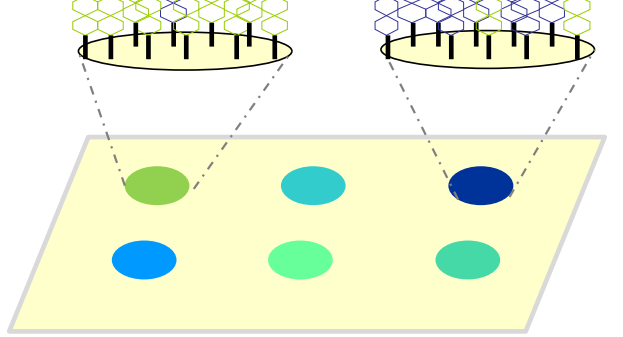

Biomimetic CoCRR array

Scheme 1. Schematic representation of a) HS and b) the CoCRR array to demonstrate its rational design by mimicking HS, based on self-assembly of two disaccharides, lactose (BB1) and sulfated lactose (BB2), at various ratios on the surface of a chip.

In practice, to construct the CoCRR array, BB1 and BB2 were prepared from $\beta$-allyl lactoside.[35] Using $\mathrm{BB} 1$ and $\mathrm{BB} 2$ at a constant total concentration, eleven solutions were prepared with $[\mathrm{BB} 1] /([\mathrm{BB} 1]+[\mathrm{BB} 2])$ ratios of $0,10,20,30,40,50,60,70,80,90$ and $100 \%$. They were then spotted on the gold surface of a prism to generate, by formation of self-assembled monolayers (SAMs), an array composed of eleven CoCRRs. For each spot, the ratio of the two building blocks anchored on the gold surface was assumed to be equal to the molar ratio in the parent solution.[38]

Firstly, the protein IFN $\gamma$ wild-type (IFN $\gamma$ WT) at $50 \mathrm{nM}$ prepared in $10 \mathrm{mM}$ HEPES buffer solution ( $\mathrm{pH}$ 7.4) was injected on the CoCRR array. Binding events were monitored in real-time by SPRi. Herein, the variation of reflectivity (expressed as $\mathrm{R} \%$ ) for each combinatorial surface was recorded versus time, giving a series of kinetic binding curves, called sensorgrams as shown in Fig. 1a. Clearly, in coherence with our previous results, [35] the affinity of IFN $\gamma$ WT to the combinatorial surfaces is dependent on $\mathrm{BB} 2 \%$ in the $\mathrm{BB}$ mixtures: the higher the sulfated $\mathrm{BB}$ ratio, the higher the IFN $\gamma \mathrm{WT}$ affinity. Notably, the protein has the highest affinity to the combinatorial surfaces composed of 100 to $90 \%$ of sulfated BB2. 
a)

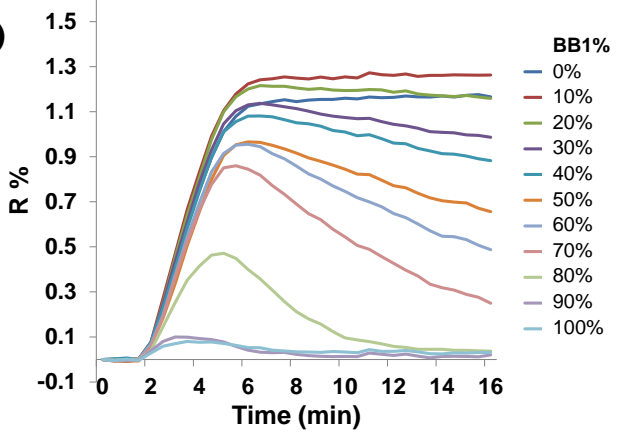

c)

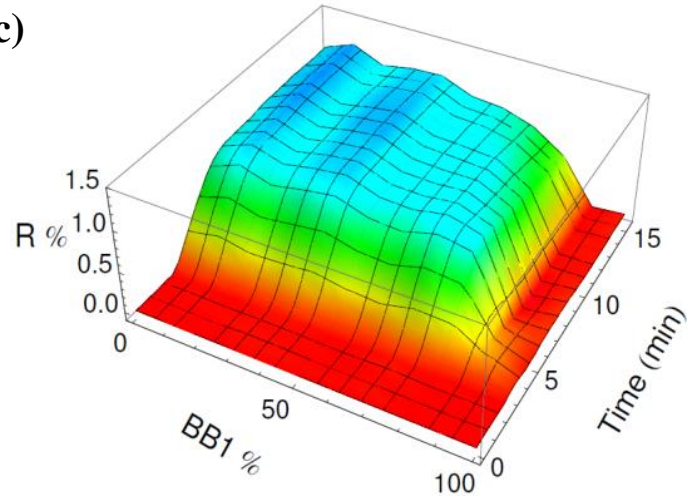

b)

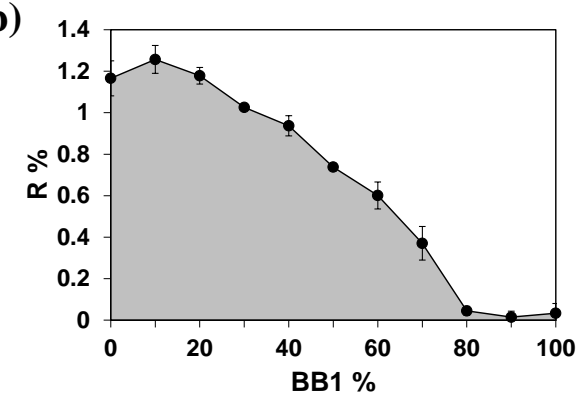

d)

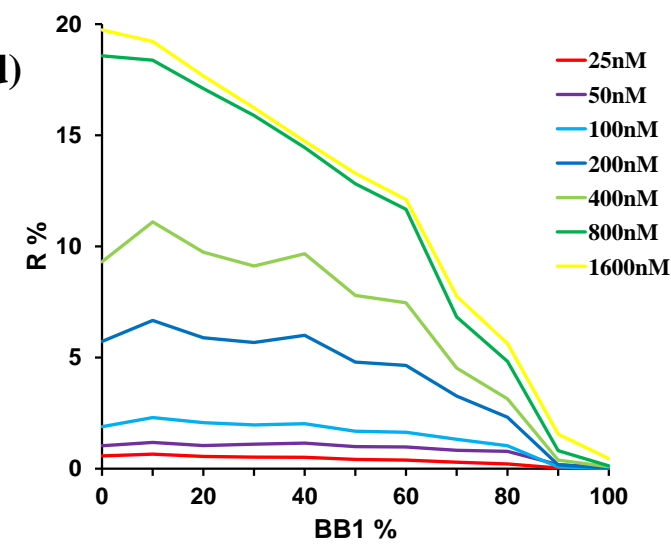

Figure 1. a) Sensorgrams showing the response of each combinatorial surface for IFN $\gamma$ WT at $50 \mathrm{nM}$. b) The pattern generated by the CoCRR array for IFN $\gamma$ WT $(50 \mathrm{nM})$, called continuous evolution profile (CEP) showing the response of each combinatorial surface for IFN $\gamma$ WT at $13^{\text {th }}$ minute after protein injection (Error bars were obtained with the four replicates of each combinatorial surface on the chip.). c) The second pattern generated for

IFN $\gamma$ WT $(50 \mathrm{nM})$ by integrating adsorption and desorption kinetics thanks to SPRi, called 3D continuous evolution landscape (CEL). Note: R\% increases from red $\rightarrow$ yellow $\rightarrow$ green $\rightarrow$ blue. d) CEPs of IFN $\gamma$ WT at different concentrations.

Based on the sensorgrams, a pattern was generated for IFN $\gamma$ WT by plotting the variation of reflectivity ( $\mathrm{R} \%$ ) versus $\mathrm{BB} 1 \%$ in the $\mathrm{BB}$ mixtures at equilibrium (13 minutes after protein injection). Notably, according to the combinatorial approach, the composition of each sensing receptor is linked and close to that of its neighbors so that each receptor provides a signal that is coherent to that of its neighbor. Thus, the series of signals generated upon interaction between combinatorial surfaces and analytes can be considered as continuous.[35] Consequently, a 2D CEP can be generated, as shown in Fig. 1b. Moreover, thanks to SPRi, we can introduce adsorption and desorption kinetics as a new discrimination parameter by adding time as the third dimension in the CEP. In this way, a timedependent recognition pattern was obtained, called 3D CEL (see Fig. 1c). Both CEP and CEL are characteristic of the protein and thus can be used as "fingerprints" for its identification based on pattern recognition. However, CEL can provide supplementary information since two analytes presenting the same relative affinity for the CoCRR array may differ in their kinetics of interaction.

Furthermore, IFN $\gamma$ WT was analyzed in a large range of concentrations: $25 \mathrm{nM}, 50 \mathrm{nM}, 100 \mathrm{nM}, 200$ nM, $400 \mathrm{nM}, 800 \mathrm{nM}$, and $1600 \mathrm{nM}$. Its CEPs at various concentrations displayed Fig. 1d. For most combinatorial surfaces, the signal intensity is proportional to the protein concentration. Interestingly, 
the shape of all the CEPs are nearly the same. Therefore, the CoCRR array has a large dynamic range, capable of detecting IFN $\gamma$ WT in the low nanomolar range before reaching saturation at high protein concentrations (> $800 \mathrm{nM})$.

Since the CoCRR array was designed to mimic biological properties of HS, it was expected that the system would show higher affinity to HSbps than to non-HSbps, even the ones having an isoelectric point (pI) above the $\mathrm{pH}$ of the running buffer (7.4). To challenge this hypothesis, the three non-HSbps Bovine Serum Albumin (BSA, $\mathrm{pI}=5.2$ ), Immunoglobulin $\mathrm{G}$ from rabbit serum $(\mathrm{IgG}, \mathrm{pI}=7.5$ ), and Cytochrome $\mathrm{C}$ from horse heart $(\mathrm{CytC}, \mathrm{pI}=10.7)$ were injected on the CoCCR array under the same experimental conditions as used for IFN $\gamma$ WT. For each protein, various concentrations were tested. Gratifyingly, the CoCRR array has not only much lower affinity for BSA and IgG, but also for the positively charged CytC (at $\mathrm{pH}$ 7.4). It was found that in the low nanomolar range none of them interacts with the CoCRR array. Only at very high micromolar range concentrations is the signal intensity of their corresponding CEP (Fig. 2 a-c) comparable to that of IFN $\gamma$ WT obtained at only 50 nM (Fig. 1b).

a)

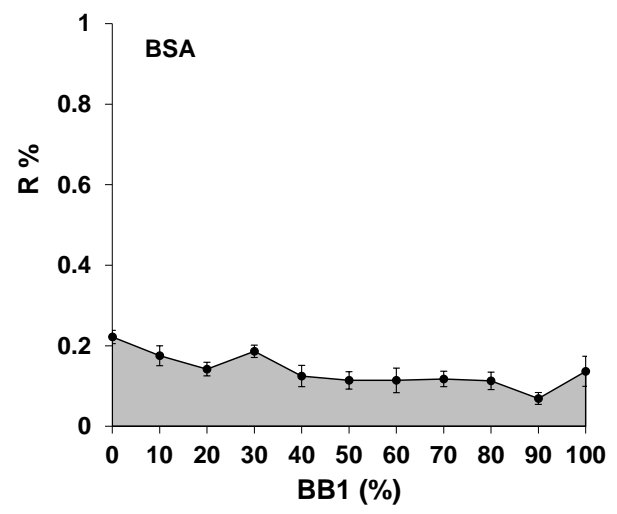

b)

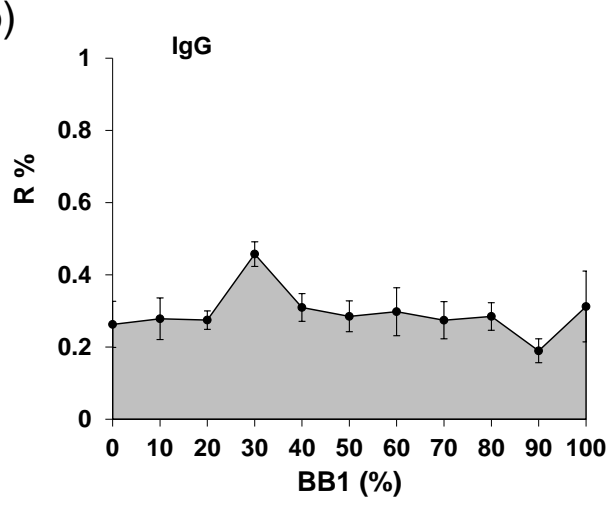

c)

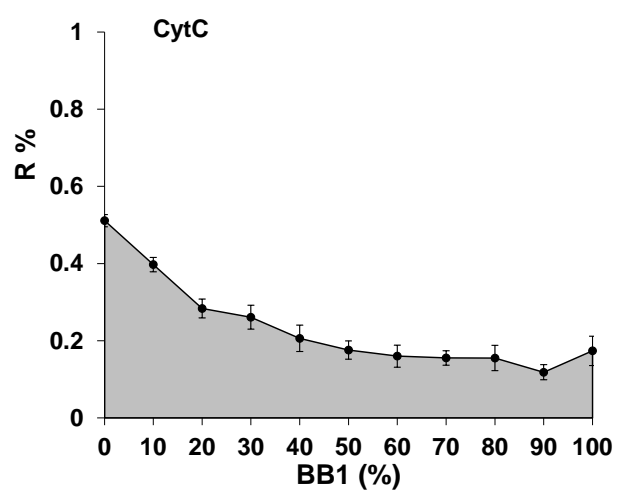

Figure 2. Sensing of non-heparan sulfate binding proteins with the CoCRR array for comparison with IFN $\gamma$ WT. CEPs of a) BSA at $20 \mu \mathrm{M}$, b) IgG at $1 \mu \mathrm{M}$, and c) CytC at $1 \mu \mathrm{M}$. Error bars were obtained with the four replicates of each combinatorial surface on the chip.

For example, Fig. 2 shows the CEPs of the three proteins generated at selected concentrations: BSA $(20 \mu \mathrm{M})$, IgG $(1 \mu \mathrm{M})$ and CytC $(1 \mu \mathrm{M})$. For negatively charged BSA and neutral IgG, the proteins bound to all the combinatorial surfaces almost in the same way due to simple non-specific adsorption. 
For the positively charged $\mathrm{CytC}$ (at $\mathrm{pH}$ 7.4), higher interaction with the combinatorial surfaces rich in negatively charged BB2 was observed, probably due to non-specific electrostatic interaction. However, a 20-fold higher concentration was required to obtain a signal intensity comparable to that of IFN $\gamma$ WT (50 nM), whose $\mathrm{pI}(10.1)$ is similar to that of CytC.

Moreover, in our previous work,[35] the CoCRR array was used to analyse two other HSbps: the $\alpha$ and $\gamma$ isoforms of the chemokine CXCL12. These two proteins can be easily discriminated from each other based on their CEPs. When compared with IFN $\gamma$, the CEP of IFN $\gamma$ was distinct from that of CXCL12 $\alpha$ but barely distinguishable from the one of CXCL12 $\gamma$. This was explained by their structures. In fact, both CXCL12 $\gamma$ and IFN $\gamma$ possess two distant HS binding domains. At least one of them is located in an unfolded part of the protein, whose flexibility could thus maximize contact points with their ligands or with low charged CoCRRs through conformational fluctuations. For a better discrimination between IFN $\gamma$ and CXCL12 $\gamma$, more BB1/BB2 mixtures and/or their mixtures with some other BBs with complementary physicochemical properties should be integrated into the CoCRR array. All these results confirm that the CoCRR array, designed to mimic the biological properties of HS, is able to discriminate not only HSbps from non-HSBPs, but also between different HSbps . In the following study, to better understand the high affinity between the CoCRR array and IFN $\gamma$ WT and in particular to investigate if the CoCRR array is able to discriminate between proteins with very similar structures, different IFN $\gamma$ mutants were designed. The carboxyl-terminal domain of IFN $\gamma$ (amino acids 124-143, AKTGKRKRSQMLFRGRRASQ) consists of two adjacent clusters of basic amino acids including domain 1 (D1) with residues 125-131 (KTGKRKR) and domain 2 (D2) with residues 137-140 (RGRR).[39] According to our previous findings both D1 and D2 are implicated in HS recognition but D1 is the predominant binding site.[36]

a)

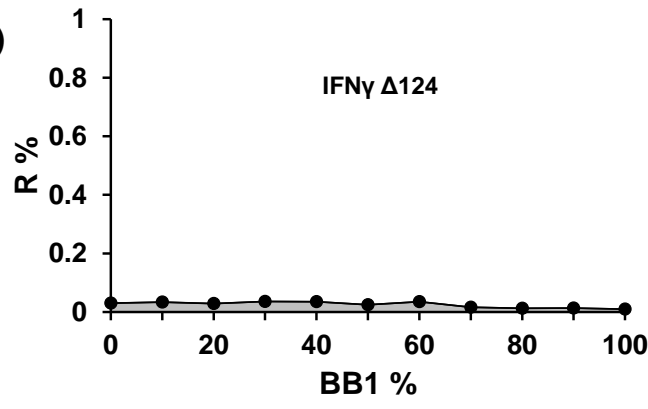

c)

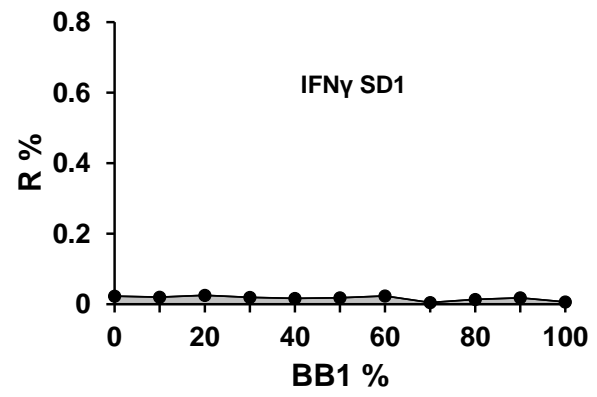

b)

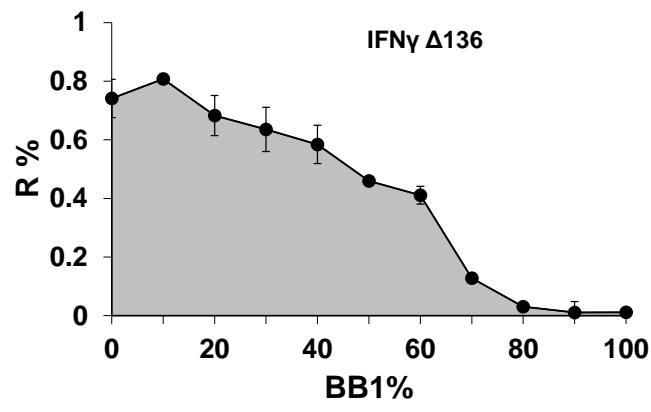

d)

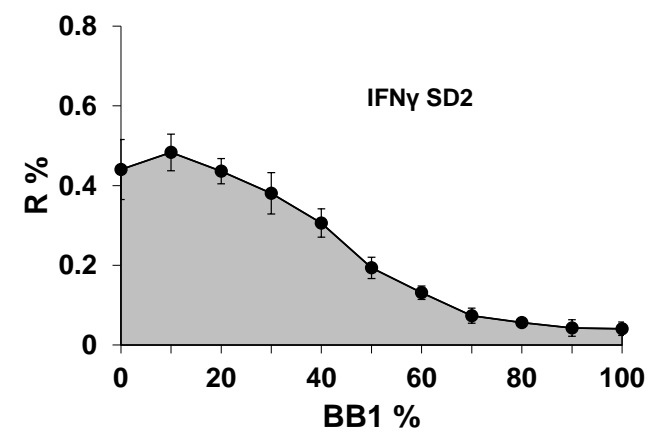


Figure 3. Analysis of IFN $\gamma$ mutants with major modifications in D1 and D2 respectively using the CoCRR array. CEPs of a) IFN $\gamma \Delta 124$, b) IFN $\gamma \Delta 136$, c) IFN $\gamma$ SD $_{1}$ and d) IFN $\gamma$ SD2. The concentration used for all the proteins was $50 \mathrm{nM}$. Error bars were obtained with the four replicates of each combinatorial surface on the chip.

In order to determine if the CoCRR array is able to differentiate between the protein IFN $\gamma$ WT and some mutants with major modifications in D1 and D2, two mutants IFN $\gamma \Delta 124$ and IFN $\gamma \Delta 136$ were used. They were obtained by deleting the terminal sequences 125-143 (both D1 and D2) and 137-143 (only D2), respectively. The two proteins were injected onto the CoCRR array at $50 \mathrm{nM}$. Their corresponding CEPs are given in Fig. $3 \mathrm{a}$ and $3 \mathrm{~b}$. For IFN $\gamma \Delta 124$, there was almost no interaction with the CoCRR array. This means that the deletion of the D1 and D2 led to the complete loss of affinity of the protein to the system (Fig.3a). It is noteworthy that there are some other clusters of basic amino acids in IFN $\gamma$ WT besides D1 and D2. However, obviously, they do not significantly contribute to the binding of the protein on the combinatorial surfaces at such a concentration. In contrast, for IFN $\gamma$ $\Delta 136$, with only the removal of the D2, the shape of its CEP was similar to that of IFN $\gamma$ WT but with lower signal intensity (Fig.3b). Taken together, the CoCRR array is able to differentiate between the three proteins. Furthermore, we intend to determine the respective importance of D1 and D2 in the interaction between the CoCRR array and IFN $\gamma$ WT. Based on these results, it is most likely that D1 is the predominant binding site since its removal led to the complete loss of affinity of the protein to the system.

To further confirm this point, the mutants IFN $\gamma$ SD1 (amino acids 124-143, A $\underline{\mathbf{S} T G S S S S S} Q M L F R G R R A S Q$ ) and IFN $\gamma$ SD2 (amino acids 124-143, AKTGKRKRSQMLF $\underline{S}$ SSSASQ) were designed by substituting all basic amino acid residues with the neutral serine in D1 and D2, respectively. For IFN $\gamma$ SD1, the CEP (Fig.3c) showed that, just like IFN $\gamma$ $\Delta 124$, there was almost no interaction with the CoCRR array despite the presence of D2. These results show that similarly to HS, the CoCRR array is extremely sensitive to major changes in D1 cluster. While for IFN $\gamma$ SD2 with major modification in D2 (Fig.3d) a significant decrease of CEP signal intensity was observed but this mutant did not completely lose affinity to the CoCRR array. Therefore, these data confirm that D1 is the predominant binding site for the interaction between the CoCRR array and IFN $\gamma$ WT. 

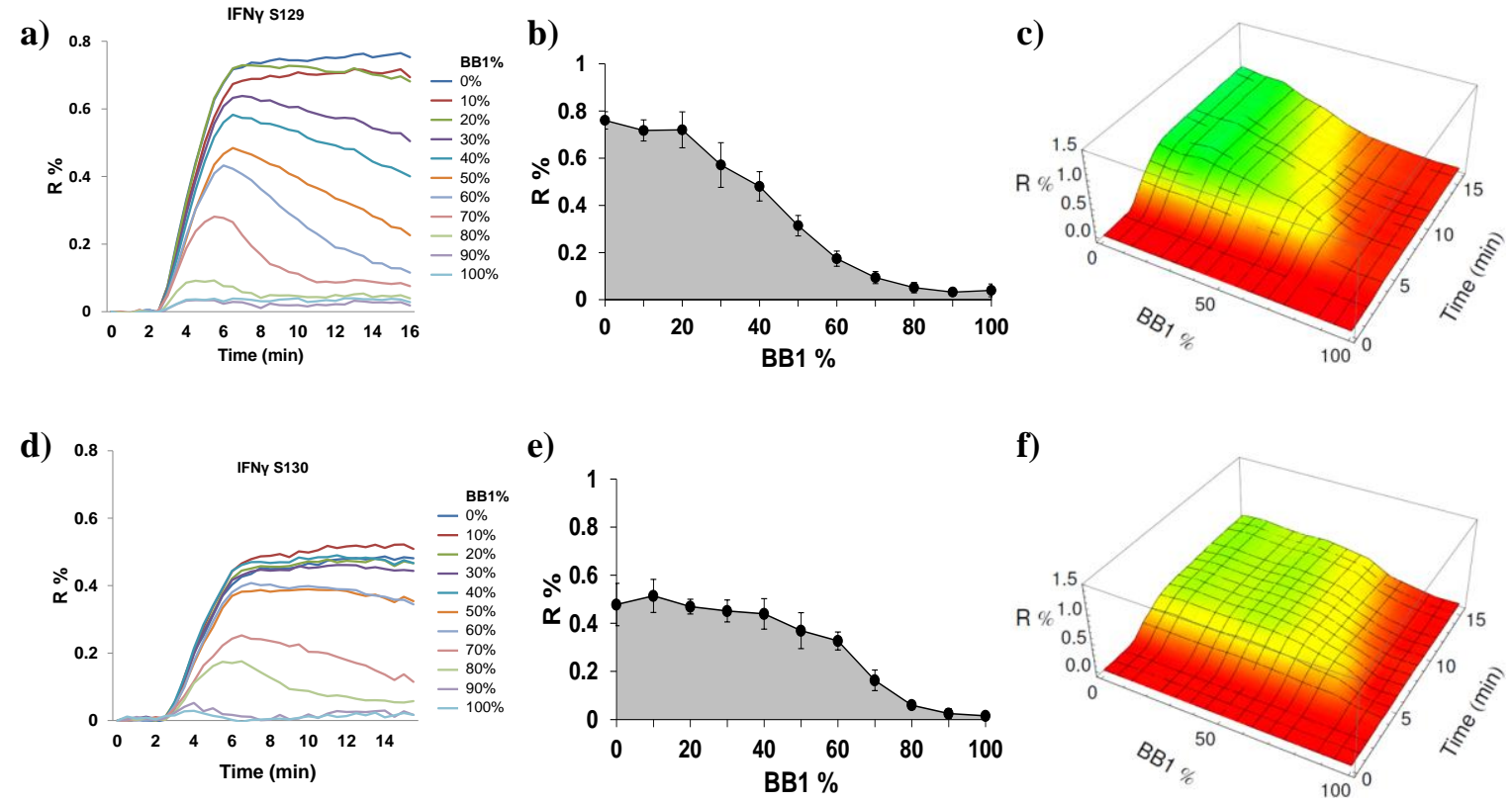

Figure 4. Sensing of the IFN $\gamma$ WT's single-point mutants with the CoCRR array. a) Sensorgrams, b) CEP and c)

CEL of IFN $\gamma$ S129, d) Sensorgrams, e) CEP and f) CEL of IFN $\gamma$ S130. Both proteins were injected on the

CoCRR array at $50 \mathrm{nM}$. Error bars for $\mathrm{b}$ and e were obtained with the four replicates of each combinatorial surface on the chip. Note: for c) and f), $\mathrm{R} \%$ increases from red $\rightarrow$ yellow $\rightarrow$ green.

Finally, we have evaluated the capability of the CoCRR array to discriminate between IFN $\gamma$ WT and its single-point mutants. For this, two single amino acid mutants in D1 were prepared, IFN $\gamma$ S129 and IFN $\gamma$ S130 in which the residues $\mathrm{R}^{129}$ and $\mathrm{K}^{130}$ were substituted with a $\mathrm{S}$ residue respectively (amino acids 124-143, AKTGKSKRSQMLFRGRRASQ and AKTGKRSRSQMLFRGRRASQ). They were subsequently injected onto the CoCRR array at $50 \mathrm{nM}$. The obtained sensorgrams, CEPs and CELs for the two mutants are shown in Fig. 4. Compared to the protein IFN $\gamma$ WT, the two mutants both have reduced affinity for the CoCRR array. Moreover, the CEP and CEL of these two single amino acid mutants are somehow different to that of IFN $\gamma$ WT. In particular, the differences are evident for their CELs, in which the kinetics of interaction were taken into account.

It is important to mention that the three dimensional structure of IFN $\gamma$ has been extensively studied. It is known that the C-terminal domain, including the two HS binding sites, is fully solvent exposed and does not adopt a specific folding. Consequently, it is not engaged in intramolecular contacts.[40] In addition, in our previous work, IFN $\gamma$ lacking parts of the (or the entire) C-terminal domain had been expressed and was shown to be correctly folded.[36] Therefore, it is known that the mutations we generated do not disturb the protein fold. We could thus conclude that the changes in binding are linked to the charge in basic amino acid residue's content in the protein $C$-terminal domain.

Therefore, these findings indicate that the CoCRR array is also capable of perceiving such subtle changes made in the $\mathrm{C}$-terminal region of the protein. To the best of our knowledge, to date there is no multisensor system that has achieved such a good selectivity with the capability to differentiate between proteins with only a single amino acid difference, based on the cross-reactivity principle and pattern recognition. 


\section{Conclusions}

In conclusion, based on our rational biomimetic methodology, the CoCRR array, prepared by using simple and easily accessible disaccharides, can mimic the biological properties of HS. Coupled with SPRi, such an array is very efficient for the analysis of HSbps with high sensitivity and selectivity. In addition, and more importantly, it is even capable of discriminating subtle differences between proteins, such as shown for IFN $\gamma$ and its single point mutants, used here as a model system. This is the first time that such a good selectivity is obtained based on cross-reactive arrays. The CoCRR arraySPRi system is reusable after regeneration. Furthermore, by introducing novel BBs with complementary physicochemical properties, the CoCRR array with improved protein binding properties could be developed. Thus, it represents a simple and promising tool for protein sensing as well as for better understanding of HS-protein interaction mechanisms. Furthermore, it could be highly useful for research on novel GAG mimetic drugs.

\section{Acknowledgment}

The authors thank greatly Labex LANEF at Grenoble (ANR-10-LABX-51-01) for supporting the PhD scholarship of Laurie-Amandine Garçon and the CEA's "Programme Transversal Nanosciences" for supporting the postdoc of Yulia Sergeeva. IRIG-SyMMES is a part of Labex ARCANE and CBHEUR-GS (ANR-17-EURE-0003) and GRAL (ANR-10-LABX-49-01).

\section{Declarations}

The authors declare no conflicts of interest.

\section{References}

1. Garçon LA, Genua M, Hou Y, Buhot A, Calemczuk R, Livache T, Billon M, Le Narvor C, Bonnaffé $D$, Lortat-Jacob $H$, Hou Y. A versatile electronic tongue based on surface plasmon resonance imaging and cross-reactive sensor arrays-a mini-review. Sensors 2017; 17:1046 .

2. Geng Y, Peveler WJ, Rotello VM. Array-based "chemical nose" sensing in diagnostics and drug discovery. Angew. Chem. Int. Ed. 2019; 58:5190-5200.

3. Margulies D, Hamilton AD. Combinatorial protein recognition as an alternative approach to antibody-mimetics. Curr. Opin. Chem. Biol. 2010; 14:705-712.

4. Baldini L, Wilson AJ, Hong J, Hamilton AD. Pattern-based detection of different proteins using an array of fluorescent protein surface receptors. J. Am. Chem. Soc. 2004; 126:5656-5657.

5. Zhou H, Baldini L, Hong J, Wilson AJ, Hamilton AD. Pattern recognition of proteins based on an array of functionalized porphyrins. J. Am. Chem. Soc. 2006; 128:2421-2425.

6. Wright AT, Griffin MJ, Zhong Z, McCleskey SC, Anslyn EV, McDevitt JT. Differential receptors create patterns that distinguish various proteins. Angew. Chem. Int. Ed. 2005; 44:6375-6378.

7. Miranda OR, You C-C, Phillips R, Kim I-B, Ghosh PS, Bunz UHF, Rotello VM. Array-based sensing of proteins using conjugated polymers. J. Am. Chem. Soc. 2007; 129:9856-9857. 
8. You CC, Miranda OR, Gider B, Ghosh PS, Kim I-B, Erdogan B, Krovi SA, Bunz UHF, Rotello VM. Detection and identification of proteins using nanoparticle-fluorescent polymer 'chemical nose' sensors. Nat. Nanotechnol. 2007;2:318-323.

9. De M, Rana S, Akpinar H, Miranda OR, Arvizo RR, Bunz UHF, Rotello VM. Sensing of proteins in human serum using conjugates of nanoparticles and green fluorescent protein. Nat. Chem. 2009; 1:461-465.

10. Miranda OR, Chen HT, You CC, Mortenson DE, Yang XC, Bunz UHF, Rotello VM. Enzymeamplified array sensing of proteins in solution and in biofluids. J. Am. Chem. Soc. 2010; 132:52855289.

11. Hewitt $\mathrm{SH}$, Wilson AJ. Protein sensing and discrimination using highly functionalised ruthenium( II ) tris(bipyridyl) protein surface mimetics in an array format. Chem. Commun. 2017; 53:12278-12281.

12. Yuan Z, Du Y, Tseng YT, Peng M, Cai N, He Y, Chang HT, Yeung ES. Fluorescent gold nanodots based sensor array for proteins discrimination. Anal. Chem. 2015; 87:4253-4259.

13. Chen S, Wei L, Chen XW, Wang JH. Suspension array of ionic liquid or ionic liquid-quantum dots conjugates for the discrimination of proteins and bacteria. Anal. Chem. 2015; 87:10902-10909.

14. Xu S, Lu X, Yao C, Huang F, Jiang H, Hua W, Na N, Liu H, Ouyang J. A visual sensor array for pattern recognition analysis of proteins using novel blue-emitting fluorescent gold nanoclusters. Anal. Chem. 2014; 86:11634-11639.

15. Xu S, Gao T, Feng X, Fan X, Liu G, Mao Y, Yu X, Lin J, Luo X. Near infrared fluorescent dual ligand functionalized Au NCs based multidimensional sensor array for pattern recognition of multiple proteins and serum discrimination. Biosens. Bioelectron. 2017; 97:203-207.

16. Xu S, Wu Y, Sun X, Wang Z, Luo X. A multicoloured Au NCs based cross-reactive sensor array for discrimination of multiple proteins. J. Mater. Chem. B 2017; 5:4207-4213.

17. Lu Y, Liu Y, Zhang S, Wang S, Zhang S, Zhang X. Aptamer-based plasmonic sensor array for discrimination of proteins and cells with the naked eye. Anal. Chem. 2013; 85:6571-6574.

18. Sun J, Lu Y, He L, Pang J, Yang F, Liu Y. Colorimetric sensor array based on gold nanoparticles: Design principles and recent advances. TrAC, Trends Anal. Chem. 2020; 122:115754.

19. Bernfield M, Götte M, Park PW, Reizes O, Fitzgerald ML, Lincecum J, Zako M. Functions of cell surface heparan sulfate proteoglycans. Annu. Rev. Biochem. 1999; 68:729-777.

20. Parish CR. The role of heparan sulphate in inflammation. Nat. Rev. Immunol. 2006; 6:633643.

21. Chiodelli P, Bugatti A, Urbinati C, Rusnati M. Heparin/heparan sulfate proteoglycans glycomic interactome in angiogenesis: biological implications and therapeutical use. Molecules 2015; 20:63426388.

22. Papy-Garcia D, Christophe M, Minh Bao H, Fernando S, Ludmilla S, Diaz Julia Elisa S, Rita RV. Glycosaminoglycans, protein aggregation and neurodegeneration. Curr. Protein Pept. Sci. 2011; 12:258-268.

23. Sasisekharan R, Shriver Z, Venkataraman G, Narayanasami U. Roles of heparan-sulphate glycosaminoglycans in cancer. Nat. Rev. Cancer 2002; 2:521-528. https://doi.org/10.1038/nrc842 
24. Bonnaffé D. Bioactive synthetic heparan sulfate and heparin derivatives: From long fragments mimetics to chimeras. Comptes Rendus Chimie 2011; 14:59-73.

25. Lubineau A, Lortat-Jacob H, Gavard O, Sarrazin S, Bonnaffé D. Synthesis of tailor-made glycoconjugate mimetics of heparan sulfate that bind IFN- $\gamma$ in the nanomolar range. Chem. Eur. J. 2004; 10:4265-4282.

26. Imberty A, Lortat-Jacob H, Pérez S. Structural view of glycosaminoglycan-protein interactions. Carbohydr. Res. 2007; 342:430-439.

27. Lortat-Jacob H, Grosdidier A, Imberty A. Structural diversity of heparan sulfate binding domains in chemokines. Proc. Natl. Acad. Sci. U.S.A 2002; 99:1229-1234.

28. Lindahl U, Li J. Interactions between heparan sulfate and proteins-design and functional implications. In: Kwang W. Jeon, editor. International Review of Cell and Molecular Biology. Academic Press; 2009. pp 105-159.

29. Kreuger J, Spillmann D, Li J, Lindahl U. Interactions between heparan sulfate and proteins: the concept of specificity. J. Cell Biol. 2006; 174:323-327.

30. Hu X, Ivashkiv LB. Cross-regulation of signaling pathways by interferon- $\gamma$ : implications for immune responses and autoimmune diseases. Immunity 2009; 31:539-550.

31. Wang D, Loo J, Chen J, Yam Y, Chen S-C, He H, Kong S, Ho H. Recent advances in surface plasmon resonance imaging sensors. Sensors 2019; 19:1266.

32. Wong $\mathrm{CL}$, Olivo $\mathrm{M}$. Surface plasmon resonance imaging sensors: a review. Plasmonics 2014; 9:809-824.

33. Genua M, Garçon L-A, Mounier V, Wehry H, Buhot A, Billon M, Calemczuk R, Bonnaffé D, Hou $\mathrm{Y}$, Livache T. SPR imaging based electronic tongue via landscape images for complex mixture analysis. Talanta 2014; 130:49-54.

34. Garçon L-A, Hou Y, Genua M, Buhot A, Calemczuk R, Bonnaffé D, Hou Y, Livache T. Landscapes of taste by a novel electronic tongue for the analysis of complex mixtures. Sens. Lett. 2014; 12:1059-1064.

35. Hou Y, Genua M, Tada Batista D, Calemczuk R, Buhot A, Fornarelli P, Koubachi J, Bonnaffé D, Saesen E, Laguri C, Lortat-Jacob H, Livache T. Continuous evolution profiles for electronic-tonguebased analysis. Angew. Chem. Int. Ed. 2012; 51:10394-10398.

36. Saesen E, Sarrazin S, Laguri C, Sadir R, Maurin D, Thomas A, Imberty A, Lortat-Jacob H. Insights into the mechanism by which interferon- $\gamma$ basic amino acid clusters mediate protein binding to heparan sulfate. J. Am. Chem. Soc. 2013; 135:9384-9390.

37. Sarrazin S, Bonnaffé D, Lubineau A, Lortat-Jacob H. Heparan sulfate mimicry: a synthetic glycoconjugate that recognizes the heparin binding domain of interferon- $\gamma$ inhibits the cytokine activity. J. Biol. Chem. 2005; 280:37558-37564.

38. Stranick SJ, Atre SV, Parikh AN, Wood MC, Allara DL, Winograd N, Weiss PS. Nanometer-scale phase separation in mixed composition self-assembled monolayers. Nanotechnology 1996; 7:438442. 
39. Vanhaverbeke C, Simorre J-P, Sadir R, Gans P, Lortat-Jacob H. NMR characterization of the interaction between the $\mathrm{C}$-terminal domain of interferon- $\gamma$ and heparin-derived oligosaccharides. Biochem. J. 2004; 384:93-99.

40. Walter MR, Windsor WT, Nagabhushan TL, Lundell DJ, Lunn CA, Zauodny PJ, Narula SK. Crystal structure of a complex between interferon- $\gamma$ and its soluble high-affinity receptor. Nature 1995; 376:230-235. 